\title{
A Note on the Hash Function of Tillich and Zémor
}

\author{
Willi Geiselmann \\ Department of Computer Science \\ Royal Holloway, University of London \\ Extended Abstract ${ }^{1}$
}

At Crypto 1994, Tillich and Zémor proposed the following hash function [1], based on the group $S L_{2}\left(I F_{2^{n}}\right)$ :

We denote the field with $2^{n}$ elements with $\mathbb{F}_{2^{n}}$. Let

$$
A:=\left(\begin{array}{cc}
\alpha & 1 \\
1 & 0
\end{array}\right) \text { and } B:=\left(\begin{array}{cc}
\alpha & \alpha+1 \\
1 & 1
\end{array}\right)
$$

be elements of $\mathrm{SL}_{2}\left(\mathrm{IF}_{2^{n}}\right)$, the group of $2 \times 2$-matrices with determinant 1 over $I F_{2^{n}}$. Let $\alpha \in I F_{2^{n}}$ denote the zero of a generating polynomial $p(x)$ of the field $I F_{2^{n}} \simeq I F_{2}[x] / p(x)$. The hash value $h \in I F_{2^{n}}^{2 \times 2}$ of a binary stream $a_{0} \ldots a_{m}$ is calculated as the product $M_{0} \ldots M_{m}$ with

$$
M_{i}:= \begin{cases}A & \text { if } a_{i}=0 \\ B & \text { if } a_{i}=1\end{cases}
$$

We analyse this hash function by embedding the two matrix rings generated by $A$ and $B$ respectively, as subrings of $I F_{2^{n}}^{2 \times 2}$. Using this technique, clashing sequences can be found by calculating discrete logarithms in the fields $I F_{2^{n}}$ or $I F_{2^{2} n}$. These clashing sequences have the special structure of very long sequences of zeros and ones; thus this "attack" can not produce reasonable collisions. Nevertheless this result exposes an additional structure of the underlying ring and thus reduces the security of this hash function.

\section{Embeddings}

Properties of the matrices $A$ and $B$ will be discussed for $A$ only. For $B$ similar relations hold. We employ the matrix representation of $I F\left(2^{n}\right)^{z}$ over $I F_{2^{n}}$ to show that

$$
V:=I F_{2^{n}} \cdot A+I F_{2^{n}} \cdot I
$$

is a field with $\left(2^{n}\right)^{2}$ elements, if $x^{2}+\alpha x+1 \in I F_{2^{n}}[x]$ is irreducible. Here, $I \in I F_{2^{n}}^{2 \times 2}$ denotes the identity matrix.

If $(x+\beta)\left(x+\frac{1}{\beta}\right)=x^{2}+\alpha x+1 \in I F_{2^{n}}[x]$ is reducible, then we define an equivalence relation on the algebra $V$ by:

1 The full paper appears in "Proceedings of Cryptography and Coding; Cirencester; 1995 " [2] 
Lemma 1. Let $M_{1}=\lambda_{1} A+\mu_{1} I, M_{2}=\lambda_{2} A+\mu_{2} I \in V$. Then

$$
M_{1} \simeq M_{2}: \Longleftrightarrow \lambda_{1} \cdot \beta+\mu_{1}=\lambda_{2} \cdot \beta+\mu_{2}
$$

defines an equivalence relation on the algebra $V$.

With this relation it is easy to show that $V / \simeq$ is a field with $2^{n}$ elements. In both the reducible and irreduclible case, $A$ and its powers can now be regarded as elements of a finite field. The main properties are summarised in the following

Theorem 2. Let $A:=\left(\begin{array}{ll}\alpha & 1 \\ 1 & 0\end{array}\right) \in S L_{2}\left(I F_{2^{n}}\right)$ and let $\hat{A}(x):=x^{2}+\alpha x+1 \in I F_{2^{n}}$ factor into $\hat{A}(x)=(x+\beta)\left(x+\frac{1}{\beta}\right)$. Then a matrix $M \in I F_{2^{n}}^{2 \times 2}$ is a power of $A$, iff the following conditions hold:

- $M=\lambda A+\mu I$ for some $\lambda, \mu \in I F_{2^{n}}$,

$-\operatorname{det}(M)=1$ and

$-M^{\text {ord }(A)}=1$.

\section{Clashing sequences}

With the results of the previous section it is very easy to find clashing sequences for the $S L_{2}$ hash function:

- Choose $C \in S L_{2}\left(I F_{2^{n}}\right)$ with a given factorization into $A$ 's and $B$ 's and a small integer $l(=3,4)$, and assume

$$
A^{i_{1}} B^{i_{2}} \ldots A^{i_{1}}=\left(\lambda_{1} A+\mu_{1} I\right) \cdot\left(\lambda_{2} B+\mu_{2} I\right) \cdot \ldots \cdot\left(\lambda_{l} A+\mu_{l} I\right)=C .
$$

- This gives 4 polynomial equations of degree $l$. Add the $l$ equations for the determinants, i.e. $\operatorname{det}\left(\lambda_{i} A+\mu_{i} I\right)=1$, and solve this system of polynomial equations over $I F_{2^{n}}$.

- Check the conditions $\left(\lambda_{i} A+\mu_{i} I\right)^{\text {ord }(A)}=1$ or $\left(\lambda_{i} B+\mu_{i} I\right)^{\text {ord }(B)}=1$. If they are not fulfilled, try again, otherwise calculate the discrete logarithms of the matrices calculated.

I have implemented this method in AXIOM and for the small example $I_{2^{23}}$ with defining polynomial $p(x)=x^{21}+x^{2}+1$, I have found lots of clashing sequences. As expected the sequences are all very long; one of the shorter ones is: $A^{79670}=$ $B^{3} \cdot A^{7} \cdot B^{69216} \cdot A \cdot B^{88234}$, where ord $(A)=2097151$ and ord $(B)=699051$.

\section{References}

1. J-P. Tillich, G. Zémor; Hashing with $S L_{2}$; Proceedings of CRYPTO'94, Y. Desmedt (Ed.), LNCS Vol 839, Springer, pp. 40-49, 1994.

2. W. Geiselmann; $A$ Note on the Hash Function of Tillich and Zémor, Proceedings of the 5th IMA Conference on Cryptography and Coding, C. Boyd (Ed.), LNCS Vol 1025, Springer, pp. 257-263, 1995. 